\title{
LOS PROCESOS INICIALES DE LECTO-ESCRITURA EN EL NIVEL DE EDUCACIÓN INICIAL
}

\section{Ana Lupita Chaves Salas'}

\author{
"Acabo de entrar a este kínder y \\ ya he aprendido a leer, inglés y a escribir" \\ Ana María, 5 años y 8 meses
}

12-3-99

\begin{abstract}
RESUMEN: El análisis de los procesos iniciales de lecto-escritura, propósito de este artículo, parte de una descripción de la función de la educación inicial y de cómo en este nivel se han incentivado dichos procesos. Posteriormente, se estudia la apropiación de la lengua escrita por parte de la niña y el niño, concebida como un proceso constructivo, interactivo y de producción cultural. Finalmente, se hacen algunas reflexiones con el fin de provocar la discusión en torno a este tema entre las personas interesadas, y así, contribuir a la transformación del nivel inicial del sistema educativo costarricense.
\end{abstract}

Palabras clave: LECTOESCRITURA/ EDUCACIÓN PREESCOLAR/ LENGUA ESCRITA/ EDUCACIÓN INICIAL/ SISTEMA EDUCATIVO COSTARRICENSE/

\section{Introducción}

Con este artículo, se aportan elementos para cuestionar las prácticas de aprestamiento que se llevan a cabo en los centros de educación inicial dirigidas a promover el desarrollo de destrezas y habilidades que les permitan a las niñas y a los niños enfrentar con éxito las distintas tareas que demanda el aprendizaje de la lectura y la escritura en el primer nivel de la Educación General Básica. Dichas prácticas responden a enfoques empiristas y conductistas del aprendizaje, los cuales, postulan que la persona aprende por simple repetición, dejando de lado que el sujeto construye el conocimiento a partir de la interacción con el medio sociocultural, las personas, los objetos, la creación de hipótesis y los intentos por comprender el mundo que lo rodea.

\footnotetext{
1 Doctora en Educación con énfasis en Investigación Educativa de la Universidad Estatal a Distancia en Costa Rica. Maestría en Educación de Adultos de la Universidad de Costa Rica. Bachiller y Licenciada en Educación Preescolar de la Universidad de Costa Rica. Directora del Instituto de Investigación para el Mejoramiento de la Educación Costarricense (IIMEC). Profesora de la Escuela de Formación Docente, Sección de Preescolar.

e-mail: jorgeurena@amnet.co.ch
} 
Las investigaciones (Norman Jackson, 1982; Chomsky, 1971; Clay, 1975; Cohn, 1981 citadas por Ruiz, Daisy, 1996) sobre los lectores naturales, es decir, niños y niñas que aprenden a leer en su hogar sin instrucción formal, coinciden en que el lenguaje escrito emerge de una necesidad de comunicarse con los demás y que el ambiente ejerce una gran influencia en el desarrollo de esta habilidad. Estos estudios llevan a cuestionar las prácticas de aprestamiento, divididas en diferentes áreas, que realizan los párvulos en los centros de educación inicial, y que, en muchos casos, representan actividades sin sentido, en las que se supone que el sujeto aprende repitiendo, de forma pasiva y mecánica.

El análisis de los procesos iniciales de lecto-escritura, propósito de este trabajo, parte de una aproximación teórica de la función de la educación inicial y del proceso que se ha seguido en torno a este tema en nuestro país. Posteriormente, se estudia la apropiación de la lengua escrita por parte de la niña y el niño, concebida como un proceso constructivo, interactivo, de producción cultural, que lleva a la reflexión y a la acción del sujeto; como una propuesta pedagógica que contribuya a la transformación, en este nivel, del sistema educativo nacional.

\section{Función de la educación inicial}

La educación inicial es el primer nivel educativo al que ingresan los niños y niñas menores de seis años. De acuerdo con los "Fines de la Educación Preescolar", que se establecen en la Ley Fundamental de Educación de 1957, éstos se dirigen a promover el desarrollo integral de la niñez durante los primeros seis años de vida. Por esta razón, el nivel tiene identidad propia, es decir, no representa únicamente una etapa de preparación para el ingreso a la escuela, por lo que se hablará de educación inicial y no de educación preescolar.

La educación del menor de seis años tiene características diferentes en relación con los otros niveles del sistema educativo, ya que atiende a una población que se encuentra en una etapa esencial para el desarrollo de la persona; al respecto, Rivera afirma:

Diversas investigaciones permiten enfatizar que el desarrollo de la inteligencia, la personalidad y el comportamiento social en los seres humanos ocurre más rápido durante los primeros años. La mayoría de las células cerebrales y las conexiones neuronales se desarrollan durante los dos primeros años y en el desenvolvimiento del cerebro -esencial para aumentar el potencial de aprendizaje - interviene no solo la salud y nutrición de los pequeños, sino factores como el tipo de interacción social y el ambiente que los rodea; los niños que sufren tensión extrema en sus primeros años 
pueden ser afectados desfavorable y permanentemente en el funcionamiento del cerebro, el aprendizaje y la memoria (1998, p. 50).

Ante la evidencia científica, es necesario fortalecer la educación del menor de seis años y de sus familias, tanto en el ámbito formal como informal, con el propósito de favorecer el desarrollo humano y la equidad social. Para tal efecto, es urgente la creación de centros infantiles de calidad en todos los estratos sociales.

El nivel de educación inicial cumple funciones económicas, sociopolíticas y pedagógicas; sin embargo, en repetidas ocasiones, se le ha dado prioridad a las dos primeras en detrimento de la función pedagógica (Denies, 1989).

Las funciones sociopolítica y económica están relacionadas, con la incorporación de la mujer al mercado laboral y con el interjuego de oferta y demanda de mano de obrał por esta razón, en un primer momento, los centros infantiles surgieron únicamente como guarderías, lugares donde se cuidaba a los infantes mientras sus madres trabajaban; posteriormente, gracias a las investigaciones científicas y al desarrollo de la psicología y la pedagogía, se toma conciencia de su valor educativo (Denies,1989).

En nuestro país, en 1997, se modifica el artículo 78 de la Constitución Política y se declara la obligatoriedad y la universalización de la educación preescolar. No obstante, aunque se han realizado esfuerzos en este sentido, existen programas para niños y niñas menores de seis años de carácter asistencial y de baja calidad, sobre todo en los estratos inferiores de la escala social. Estos programas dan énfasis a la función económica y sociopolítica del nivel, en detrimento de la función pedagógica. Al respecto, Clarke-Stewart (1987, citado por León, 1992), demuestran en sus investigaciones, que una educación de baja calidad tiene consecuencias negativas en el desarrollo de los infantes. En este mismo sentido, Peralta argumenta:

Una mala educación inicial convencional o no convencional, puede lesionar seriamente a los niños y sus familias, tanto en sus posibilidades presentes como futuras, por lo que no se trata de desarrollarla de cualquier manera, sino salvaguardando niveles de calidad básicos para asegurar la inversión. Más adelante agrega: ... aumentos de cobertura en educación inicial sin estar unido a criterios de calidad esenciales, no se justifican, razón por la cual debe velarse porque esta última esté siempre presente" (1993, p. 1).

\footnotetext{
${ }^{2}$ Para ampliar sobre la función sociopolítica y económica, revisar Chavarría, Ma. Celina, 1993
} 
La función pedagógica es la más importante en el nivel inicial, pues se ocupa de optimizar el desarrollo integral del infante, ya que considera los aspecto socioafectivo, psicomotor, cognoscitivo y nutricional, tomando como punto de partida la familia, primer agente educativo del contexto sociocultural que rodea al niño. Denies (1989) indica que a esta función se le ha adjudicado, tradicionalmente, la tarea de preparar al párvulo para la escuela primaria, de ahí el nombre que se le ha dado a este nivel: educación preescolar. Desde esta perspectiva tradicional, muchas veces se trabaja intensamente el aprestamiento, sin tomar en cuenta que el sujeto construye el conocimiento a partir de la interacción con las personas, los objetos, la creación de hipótesis y su esfuerzo por comprender el mundo que lo rodea. Por otra parte, la función pedagógica del nivel inicial es más amplia.

La función pedagógica incluye, además, una dimensión socializadora que contribuye con la construcción del ser social del párvulo, una dimensión preventiva que se encarga de evitar los efectos negativos (deficiencias o carencias afectivas, intelectuales, motrices, orgánicas) que sufren los niños y las niñas como consecuencia de la marginación socioeconómica o de otras causas; y la función recuperatoria, encargada de detectar alteraciones funcionales $u$ orgánicas que afecten el aprendizaje. Todo esto, con el fin de iniciar un proceso de intervención que ayude a superar problemas que puedan incidir negativamente en el desarrollo integral del infante (Denies, 1989, p. 5 y 6).

Desde esta perspectiva, es fundamental que el nivel de educación inicial cumpla con todas las funciones que se le han asignado, pues son numerosos los estudios que indican los beneficios de esta para la niñez y para la sociedad en general.

Myers afirma que: "Los descubrimientos científicos en una serie de áreas han demostrado que los programas orientados a los niños pequeños pueden acarrear grandes beneficios, no sólo en términos individuales e inmediatos sino también en términos sociales y económicos a lo largo de toda su vida en cuanto a sus habilidades para contribuir a la familia, la comunidad y la nación." (1995, p. 3).

Por su parte, en Estados Unidos, se realizó un estudio con niños y niñas de tres y cuatro años, todos provenientes de barrios de bajos ingresos. Estos párvulos fueron divididos en dos grupos al azar. El primero tuvo una educación preescolar de calidad y el otro no. Los subgrupos se evaluaron periódicamente hasta que los participantes cumplieron veintisiete años de edad. La investigación concluyó que las personas que asistieron a programas de educación inicial de calidad, disfrutaron de "un nivel de escolaridad significativamente superior; salarios significativamente más altos; mayor probabilidad de tener vivienda propia; y menor dependencia de los servicios sociales" (Schwenhard, et al, 
1993 citado por Rivera, 1998).

Otros estudios que comparan a párvulos con y sin educación temprana en nueve países en vías de desarrollo, señalan, entre otras ventajas de la educación inicial, las siguientes:

- el acceso a intervenciones tempranas suele tener efecto positivo sobre el progreso y desempeño escolar, con descensos sustantivos en las tasas de deserción y repetición;

- los niños pobres pueden beneficiarse más de los programas multifacéticos que sus pares beneficiados (R. Myers, 1996 citado por Rivera, 1998, p. 50).

En este contexto, es preciso que los programas de educación inicial sean de calidad y accesibles a todos los sectores de la sociedad, ya que es incuestionable la importancia de este nivel educativo para la formación del ser humano.

\section{Tendencias pedagógicas de los procesos iniciales de lectoescritura}

En Costa Rica los primeros centros de educación inicial surgieron en 1878, con las "escuelas para párvulos" de carácter religioso (Ugalde, 1983).

La Ley General de Educación Común, aprobada en 1886, contempla la creación de las "escuelas especiales"; entre ellas, "uno o más jardines de infantes en las capitales de provincia" (Dengo, 1998). Sin embargo, este aspecto de la ley no se concreta. Es hasta el año 1926 que se funda el primer jardín de infantes oficial en la ciudad de San José: la Escuela Maternal Montessoriana, ubicada en el Edificio Metálico, la cual dio inicio con dos secciones y desarrolló un programa educativo, artístico y de salud para los párvulos de sectores económicamente desfavorecidos. Fue dirigido por María Isabel Carvajal (Carmen Lyra), quien había estudiado la Pedagogía Científica de María Montessori en Europa (18701952).

El método montessoriano impulsa principios educativos como la libertad, la actividad y la autonomía; le da gran importancia a la autoeducación y al docente como guía del proceso de enseñanza y aprendizaje, y propone material didáctico autocorrector que contribuye a lograr una "educación sensorial completa y graduada" (Ugalde, 1983); para Montessori (1870-1952), los materiales educan los sentidos del niño y la niña, puesto que estos son "la base del juicio y del raciocinio" (Rojas, 1998, p. 37). 
En relación con "el método para enseñar a escribir", Montessori enfatiza que el punto esencial radica en la preparación indirecta de las habilidades motoras del sujeto y recomienda algunos ejercicios previos: dibujar el contorno y rellenar figuras geométricas; tocar las letras del alfabeto en lija, una y otra vez, con los dedos índice y medio de la mano derecha, mientras la maestra pronuncia su sonido; y componer palabras con un alfabeto movible. Con estos ejercicios preparatorios, el niño y la niña se han iniciado, también, en la lectura. (Montessori, 1939)

Para esta autora, dado que la lengua española goza de una relación fonema / grafema: basta con pronunciar claramente los diferentes componentes de una palabra (por ejemplo m-a-n-o), para que el niño, cuyo oído está ya educado, pueda reconocer uno por uno, los sonidos componentes. Entonces mira en el alfabeto movible qué signos corresponden a cada sonido, y los pone uno junto a otro, componiendo así la palabra. (Montessori, 1939, p.144-145).

El aporte de este método en el contexto nacional fue valioso, puesto que se impulsa una concepción activa del aprendizaje; sin embargo, no se evolucionó desde el punto de vista epistemológico, ya que al estar influenciado por el empirismol se considera que "la experiencia sensorial es la fuente de los conocimientos y todo saber se fundamenta en la experiencia" (Rojas, 1998, p. 23). Al respecto, Rojas indica:

Estas lagunas en la concepción de cómo conocemos van a traducirse en lagunas didácticas y educativas...someten a los alumnos a gran cantidad de experiencias y "estímulos", sin importar si estos van acordes con las estructuras, capacidad y esquemas del estudiante. (1998, p. 22).

Según esta posición, el sujeto recibe el conocimiento del objeto y del medio a través de los sentidos, y de la repetición de las experiencias.

Años después, en 1933, Emma Gamboa funda el Kindergarten de la Escuela de Aplicación de la Escuela Normal, siguiendo los principios de la "escuela activa", y como resultado de los planes de estudios para la formación de maestros y maestras, que enfatizaban las corrientes de la "escuela nueva" o "escuela activa", con influencia de "pedagogos norteamericanos (Dewey y Kilpatrick) y europeos (Kerschesteiner, Claparède, Ferrèire, Decroly). (Dengo, 1998, p. 145).

\footnotetext{
${ }^{3}$ Para una discusión más amplia sobre el Empirismo consultar: Rojas, Maricela (1998) 21-28 pp
} 
La "escuela activa" toma en cuenta los intereses del niño y la niña, respeta las diferencias individuales, desarrolla actitudes y aptitudes para el aprendizaje, parte del juego natural, emplea materiales tridimensionales, favorece un clima de libertad y autonomía; y le da al docente el rol de facilitador o guía de los procesos de enseñanza y aprendizaje (Rojas, 1998).

Durante este periodo histórico, se introdujo el método de enseñanza de lectoescritura de Decroly: método global o ideovisual, y la organización del currículo en centros de interés (Dengo, 1998, p. 145).

Ovideo Decroly (1871-1932) propuso una metodología de integración de ideas asociadas a partir de los intereses y de la realidad que rodea al niño y la niña. Esta metodología consideraba que la vida psíquica es "una totalidad" dentro de la cual se perciben las estructuras organizadas, de ahí que propone los "centros de interés" como forma de trabajo escolar, siguiendo tres etapas: observación, asociación y expresión

Este autor concibió la lectura como un acto "global" e "ideo-visual" donde lo fundamental es reconocer globalmente las palabras $u$ oraciones, para luego analizar las partes que las componen; a la vez, aboga por comenzar este proceso con unidades significativas para el párvulo. Este método emerge como reacción contra el método sintético (fonético), que Decroly consideró mecanicista, pues pensaba que "no importa cuál sea la dificultad auditiva de lo que se aprende, puesto que la lectura es una tarea fundamentalmente visual" (Ferreiro y Teberosky, 1982, p. 20).

Dentro del enfoque decrolyano se le dio gran importancia a la afectividad en el desarrollo de la personalidad y al trabajo en grupo; se creía que la niñez debía ser el centro de la escuela y la "Escuela para la vida y por la vida". (Torres S, 1996; Ugalde, 1983) Se crean una serie de juegos educativos para el desarrollo de las facultades perceptivas, los que se clasifican en: juegos visuales, juegos visomotores, juegos motores y auditivos motores, juegos de iniciación a la aritmética, juegos que se refieren a la noción del tiempo, juegos de iniciación a la lectura y juegos de gramática y comprensión de lenguaje. Todos estos juegos y los materiales decrolyanos, son la base de muchos de los recursos gráficos que se utilizan en las actividades de apresto en los centros de educación inicial en la actualidad (Peralta, 1993).

A partir de la influencia de la "escuela nueva", la educación inicial en Costa Rica empieza a crecer. Para 1935, se habían creado únicamente nueve centros infantiles oficiales y cuatro semioficiales.

\footnotetext{
${ }^{4}$ Sobre los centros de interés de Decroly, consultar Torres S, 1996 pp191-201.
} 
En 1960, la Universidad de Costa Rica (UCR) funda la Escuela Nueva Laboratorio, bajo la filosofía de la "escuela activa", impulsada por la Dra. Emma Gamboa. Desde su inicio, se trabaja con dos secciones de educación preescolar, un grupo con niños de cinco años y otro con niños de seis años. En 1966 la UCR ofrece el primer plan de estudio para la formación de maestros con énfasis en preescolar, siguiendo el planeamiento curricular de los "Centros de Interés" (Solís, 1992).

La "escuela nueva" definitivamente brinda grandes aportes a la educación costarricense; a pesar de ello, se le sigue dando mucha importancia a las habilidades perceptivas del niño y la niña para conocer, sin tomar en cuenta "...la competencia lingüística del niño y sus capacidades cognoscitivas." (Ferreiro y Teberosky 1982, p. 21). Como puede notarse, no se produce un cambio "...en lo que respecta a la concepción epistemológica del cómo conoce el niño" (Rojas, 1998, p. 37).

Por otra parte, el método ecléctico para la enseñanza de la lectoescritura, que consiste en integrar diferentes aspectos de los métodos sintéticos y analíticos $\mathbf{a}$ ha tenido gran influencia en nuestro país. Para la aplicación de este, es necesario partir de un diagnóstico previo y tomar en cuenta las diferencias individuales de las niñas y los niños, con el fin de iniciar el aprestamiento dirigido a "crear en cada niño un gran deseo de aprender" (Chacón, 1974 , p. 8) y a la vez, propiciar el desarrollo de las destrezas necesarias, para iniciar con éxito el aprendizaje formal de la lectura y la escritura. Esta función se le asigna sobre todo al nivel de educación inicial, y en consecuencia, a partir de la década de los setenta, la Universidad de Costa Rica promueve el planeamiento por destrezas para el nivel "preescolar", con el fin de que se atiendan las siguientes áreas:

- Cognoscitiva: percepción y discriminación visual, auditiva, táctil, gustativa; y desarrollo del lenguaje.

- Socioafectiva: integración del medio social, afirmación del yo, independencia, formación de sentimientos, expresión de sentimientos

- Motriz: coordinación motriz gruesa y coordinación motriz fina (Ugalde, 1983, p.144153).

Cada uno de los apartados de las áreas, detalla minuciosamente las destrezas que se deben desarrollar en los niños y las niñas para que, al ingresar a primer grado, estén preparados para el aprendizaje formal de la lecto-escritura. Sobre esto, Ruiz afirma:

\footnotetext{
${ }^{5}$ Si se desea profundizar en el método ecléctico revisar Chacón, Nora de (1974)
} 
El programa de apresto para la lectura se fundamenta también en un esquema conductista. Este marco conceptual descompone una jerarquía de destrezas en conductas observables, desde la más sencilla a la más compleja al asumir que para poder leer es fundamental reconocer primero las palabras, letras y sonidos. Al tener las letras y palabras diferentes tamaños, forma, dirección, posición, detalles, orden y sonidos, el desarrollo de la habilidad perceptual visual y auditiva recibe la mayor atención en la etapa de "preparación" o " apresto" para la lectura. El énfasis por lo tanto, se concentra en el desarrollo de las habilidades físicas-visoespaciales las cuales son necesarias, pero quizás no son las más importantes para leer, como lo demuestran los estudios realizados con los lectores naturales." (1996, p. 46)

El método ecléctico para la enseñanza de la lectoescritura da gran importancia al aprestamiento para el desarrollo de destrezas y habilidades perceptivas de parte del niño y la niña, sin tomar en cuenta que este "...es un sujeto que aprende básicamente a través de sus propias acciones sobre los objetos del mundo, y que construye sus propias categorías de pensamiento al mismo tiempo que organiza su mundo" (Ferreiro y Teberosky, 1982, p. 29).

En los últimos años, el enfoque constructivista ha tenido alguna influencia en la educación costarricense, y así se evidencia en el "Programa de Estudio del Nivel de Transición de la Educación Preescolar", del Ministerio de Educación Pública, (1996); sin embargo, en la práctica pedagógica, este cambio aún no se ha producido de una manera generalizada.

En este recorrido histórico sobre los métodos de enseñanza de la lecto-escritura que han tenido mayor influencia en nuestro país, sobresale un sujeto que aprende como receptor de un conocimiento proporcionado desde afuera, donde los protagonistas son el educador y el método, y no el sujeto que conoce, de ahí el énfasis que se le ha otorgado al aprestamiento como etapa básica de preparación para iniciar el aprendizaje formal de la lecto-escritura.

En este artículo, se plantea una visión diferente, desde un enfoque constructivista, donde el niño y la niña son protagonistas de su propio proceso de conocimiento. Él y ella ingresan al centro infantil con un bagaje de conocimientos que han adquirido en su hogar y en su comunidad, y a partir de estos, el docente brinda experiencias auténticas de lectura y de escritura que les permiten a los párvulos construir colectivamente el conocimiento de la lectoescritura. 


\section{Construcción del conocimiento y del lenguaje}

Las últimas décadas de nuestra era han sido testigo de numerosos hallazgos que nos dicen cómo los infantes se apropian del código escrito; entre estos, la teoría cognitiva de Jean Piaget (citado por Papalia y Wendkors, 1997) y la sociocultural, de Lev Vigotsky (1978).

Según Piaget, la niña y el niño construyen el conocimiento mediante la interacción con el mundo que lo rodea. En este proceso, se siguen una serie de etapas que están relacionadas con las capacidades mentales que posee el sujeto para organizar la información que recibe del medio. Para este teórico, durante los dos primeros años de vida, el ser humano inicia su conocimiento del mundo por medio de la experiencia sensorial y la actividad motriz.

Entre los dos y siete años de edad, aproximadamente, los infantes se encuentran en la etapa preoperatoria; su pensamiento es simbólico, es decir, comienzan a generar ideas y solucionar problemas a través de las representaciones mentales que se demuestran en el lenguaje, el juego simbólico y la imitación diferida; en este proceso se le asigna un significado a un símbolo. El símbolo más usado es la palabra hablada o escrita. Al respecto, Papalia y Wendkors indican:

Saber los símbolos de las cosas ayuda a pensar en ellas y en sus cualidades, a recordarlas y hablar sobre ellas, sin necesidad de tenerlas al frente. Ahora los niños pueden utilizar el lenguaje para representar cosas y hechos ausentes que no tienen lugar en el presente. Ahora pueden aprender no solo mediante los sentidos y la acción sino mediante el pensamiento simbólico, no solo al actuar sino, además, por las consecuencias de sus acciones. (1997, p. 325).

En el proceso de interacción con el medio social y físico, el infante recibe los estímulos, los transforma mediante el proceso de asimilación, los interpreta de acuerdo con sus esquemas mentales y construye su concepción de mundo al transformar las imágenes estáticas en imágenes activas por medio del lenguaje, el juego, el dibujo y la imitación. El pensamiento del párvulo surge, entonces, a través de la acción, a partir de la cual interioriza ciertas imágenes que posteriormente, aprenderá que tienen correspondencia con un nombre, y de esa manera, se origina el lenguaje, "...para Piaget, el lenguaje, como instrumento de expresión y comunicación, es susceptible de llegar a ser el instrumento privilegiado del pensamiento, en especial cuando el niño va pasando del pensamiento concreto al abstracto" (Gómez, M; Villareal, B, González, L; López, L y Jarillo, R, y otras:1995, p. 48).

Piaget habla de dos tipos de lenguaje: privado (egocéntrico) y social. El lenguaje privado está dirigido hacia sí mismo, con el fin de que el pequeño tenga control de sus 
acciones. El lenguaje social busca la comunicación con otros y se concreta con la aparición del diálogo. Conforme crece el lenguaje, evoluciona la construcción del espacio, del tiempo y de la causalidad, lo que contribuye a que el niño y la niña ubiquen sus acciones en el presente, pasado o futuro, y a la vez, organicen sus relatos en una secuencia lógica (Papalia y Wendkors, 1997).

Desde esta perspectiva, el lenguaje oral del infante contribuirá al aprendizaje de la lengua escrita en tanto sea utilizado como una forma de comunicación con el medio social (Gómez, M; Villarreal, B, González, L; López, L y Jarillo, R, 1995).

Por su parte, Vigotsky (1978) planteó el fundamento epistemológico de su teoría indicando que "...el problema del conocimiento entre el sujeto y el objeto se resuelve a través de la dialéctica marxista (S-O), donde el sujeto actúa (persona) mediado por la actividad práctica social (objetal) sobre el objeto (realidad) transformándolo $y$ transformándose a sí mismo" (Matos, 1996, p. 4). Es decir, se da una relación dialéctica entre sujeto y objeto. En este proceso de conocimiento son esenciales el uso de instrumentos socioculturales, especialmente de dos tipos: las herramientas y los signos. Las herramientas producen cambios en los objetos y los signos transforman internamente al sujeto que ejecuta la acción. Los signos son instrumentos psicológicos producto de la interacción sociocultural y de la evolución, como el lenguaje, la escritura y el cálculo, entre otros (Barquero, 1996).

Según esta teoría, el párvulo, al entrar en contacto con la cultura a la que pertenece, se apropia de los signos que son de origen social para, posteriormente, internalizarlos. Vigotsky indica "...el signo siempre es inicialmente un medio de vinculación social, un medio de acción sobre los otros y solo luego se convierte en un medio de acción sobre sí mismo" (1978, p. 141).

La niña y el niño se van apropiando de las manifestaciones culturales que tienen un significado en la actividad colectiva, es así como "...los procesos psicológicos superiores se desarrollan en los niños a través de la enculturación de las prácticas sociales, a través de la adquisición de la tecnología de la sociedad, de sus signos y herramientas, y a través de la educación en todas sus formas" (Moll, 1993, p. 13).

Vigotsky considera que el momento más significativo en el desarrollo del niño y de la niña, es cuando convergen el lenguaje y la actividad práctica, pues inicialmente, eran dos líneas de desarrollo totalmente independientes, "en un momento dado se unen y el lenguaje se vuelve racional y el pensamiento verbal. El desarrollo que hasta ahora era biológico se vuelve sociohistórico ya que por medio del lenguaje racional, la sociedad inyecta en el 
individuo las significaciones que ha elaborado en el transcurso de su historia" (Morales, 1990, p. 11).

De acuerdo con esta teoría, el lenguaje se da, en un principio en el nivel social, luego es egocéntrico, y más adelante, interiorizado (Vigotsky, 1978). Cuando el párvulo inicia la comunicación verbal, el lenguaje sigue a las acciones, es provocado y dominado por la actividad; en estadios superiores surge una nueva relación entre la palabra y la acción, ya que el lenguaje guía, determina y domina el curso de la acción y aparece su función planificadora, de tal manera que el lenguaje es esencial para el desarrollo cognoscitivo del infante (Morales, 1990).

Vigotsky (1978) considera que el significado de la palabra es clave para estudiar las relaciones internas del discurso y del pensamiento. Para él, es fundamental el papel del sentido y el significado en el desarrollo de la percepción en los niños y las niñas, así como los usos cognitivos de los signos y las herramientas, el desarrollo de la escritura y el juego, al cual le dio gran importancia para la interiorización y apropiación del ambiente durante los primeros años de vida.

El concepto vigotskiano que tiene mayor aplicabilidad en el campo educativo es la zona de desarrollo próximo (ZDP). Este concepto "designa las acciones del individuo que al inicio él puede realizar exitosamente sólo en interrelación con otras personas, en la comunicación con éstas y con su ayuda, pero que luego puede cumplir en forma totalmente autónoma $y$ voluntaria" (Matos, 1996, p. 8).

Vigotsky planteaba dos niveles de desarrollo en los niños: el nivel actual de desarrollo y la zona de desarrollo próximo, la que se encuentra en proceso de formación y es el desarrollo potencial al que el infante puede aspirar.

Este concepto es básico para los procesos de enseñanza y aprendizaje, pues el educador y la educadora deben tomar en cuenta el desarrollo del infante en sus dos niveles: el real y el potencial, para así promover niveles de avance y autorregulación mediante actividades de colaboración.

Los estudios de Piaget (citado por Papalia y Wendkors, 1997) y Vigotsky (1978), aunque presentan algunas diferencias teóricas, coinciden en que el conocimiento se da mediante un proceso constructivo del sujeto en interacción con el medio, lo que implica un cambio epistemológico que concibe al sujeto y al objeto como entes activos. Desde esta perspectiva, son cuestionables las bondades de los métodos tradicionales para la enseñanza de la lecto-escritura, puesto que "El método (en tanto acción específica del medio) puede ayudar o frenar, facilitar o dificultar, pero no crear aprendizaje. La obtención de conocimiento 
es un resultado de la propia actividad del sujeto" (Ferreiro y Teberosky, 1982, p. 32). El niño y la niña no esperan pasivamente que se les enseñe el lenguaje sino que tratan de comprenderlo, formulan hipótesis y crean su propia gramática con base en la información que le provee el contexto sociocultural en que se desenvuelven.

\section{Nuevas perspectivas pedagógicas en el aprendizaje de la lectura y la escritura}

Con base en las teorías de Piaget y de Vigotsky, se han realizado diversas investigaciones y se han formulado nuevas propuestas pedagógicas sobre el aprendizaje de la lectoescritura, entre ellas: la psicogenésis de la lengua escrita de Emilia Ferreiro (1983), las funciones lingüísticas de Halliday (1986) y el enfoque psicolingüístico de Kenneth y Yetta Goodman (1989 y 1993).

Ferreiro (1994), en sus investigaciones sobre el proceso de apropiación de la lengua escrita, encuentra que los niños y las niñas pasan por una serie de niveles y subniveles en este proceso de aprendizaje y que al ingresar a la escuela, ya tienen ciertas concepciones sobre la escritura; es decir, que desde edades muy tempranas, los párvulos tratan de explicarse la información escrita que les llega de diversas procedencias: empaques de galletas, refrescos, periódicos, libros y otros. El proceso de aprendizaje de la lecto-escritura interviene en gran medida el contexto sociocultural y la función social que tiene la lengua escrita para comunicar significados, ya que, por medio de ella, se trasmite todo tipo de conocimientos, creencias y valores. M.A.K. Halliday (1986) menciona siete categorías de funciones lingüísticas que se desarrollan en el contexto social y que aplican tanto a la lengua oral como a la lengua escrita:

- Instrumental. Es el lenguaje que se utiliza para satisfacer necesidades.

- Regulatoria. Es el que se usa para controlar la conducta de otros

- Interaccional. Se refiere al lenguaje para mantener y establecer relaciones sociales.

- Personal. Es aquel que permite expresar opiniones personales

- Imaginativa Permite expresar lo que imaginamos y creamos

- Lenguaje heurístico. Nos permite crear información y respuestas acerca de diferentes cosas que se desean conocer.

- Lenguaje informativo. Es el que permite comunicar información

\footnotetext{
${ }^{6}$ Para mayor información sobre la psicogénesis de la lengua escrita consultar, Ferreiro, 1997
} 
Es otras palabras, en la apropiación de la lengua escrita es fundamental el contexto sociocultural y el uso funcional que le dé el niño al lenguaje para comunicar significados; por tal razón, es necesario que las educadoras, los educadores y otros adultos que interactúan con las niñas y los niños, promuevan en ellos la capacidad comunicativa en todas sus formas, lo que les permitirá la socialización de sus actos, la integración con su cultura y el conocimiento del mundo.

Numerosas investigaciones (Norman Jackson, 1982, Chomsky, 1971; Clay, 1975; Cohn, 1981 citadas por Ruiz, 1996) sobre los lectores naturales, es decir, niños y niñas que aprenden a leer en su hogar sin instrucción formal, coinciden en que el lenguaje emerge de una necesidad de comunicarse con los demás, y que el ambiente ejerce una gran influencia en el desarrollo de esta habilidad. Estos ambientes se caracterizan por la interacción verbal que se da entre los miembros de la familia, donde el infante tiene acceso a todo tipo de materiales para la lectura y escritura, y donde la lectura es una actividad cotidiana por la que los adultos manifiestan interés; asimismo, responden a la curiosidad de los párvulos sobre el lenguaje escrito y a su esfuerzo por interpretarlo, de tal manera que "...la habilidad para leer no emerge de un vacío sino que se fundamenta en el conocimiento preexistente del niño sobre el lenguaje, y se construye mediante un proceso dinámico en el cual interactúan y se apoyan los cuatro procesos del lenguaje: escuchar, hablar, escribir y leer" (Ruiz, 1996, p. 43).

Todos estos aportes teóricos indican que el conocimiento sobre el lenguaje escrito se construye mediante la participación activa del infante en el contexto natural y cotidiano, al utilizarlo de una manera funcional con sentido y significados reales.

Otro aporte importante es la propuesta de Kenneth y Yetta Goodman (1989,1993), Ellos construyen una propuesta pedagógica para el aprendizaje de la lectura y la escritura (Filosofía del Lenguaje Integral) que emerge de los estudios citados anteriormente y de las investigaciones que realizaron ambos sobre los procesos de apropiación de la lectura en salas de clase con estudiantes de diferentes niveles educativos y de diversos grupos étnicos en los Estados Unidos.

La filosofía de Lenguaje Integral no es un método para enseñar a leer y a escribir sino que es una nueva concepción de lenguaje y de ser humano en interacción, donde maestros y alumnos tienen un papel protagónico en el proceso de enseñanza y de aprendizaje como investigadores de contextos socioculturales en busca del conocimiento de las diferentes realidades. Esta propuesta se basa en las siguientes premisas: el lenguaje sirve para organizar el pensamiento, para aprender, comunicar y compartir experiencias con los demás; el desarrollo cognoscitivo y lingüístico son totalmente interdependientes: el pensamiento 
depende del lenguaje y el lenguaje del pensamiento; el aprendizaje es la construcción social del conocimiento, el aprendizaje y la enseñanza son procesos dialógicos, y el aprendizaje del lenguaje es fácil si se parte de los contextos socioculturales de los estudiantes (Goodman, 1993 y Arellano, 1994).

Mediante el lenguaje, se accede al conocimiento, por eso, es de vital importancia que el ser humano se apropie de la lengua escrita; pues se lee y se escribe para aprender y conocer el mundo.

Goodman (1993) hace una crítica al sistema escolar: según él, se pretende que las personas aprendan el lenguaje en pequeños fragmentos, de lo particular a lo general, sin propósito ni significado para el sujeto y fuera de contexto. Censura el uso de textos y el llenar hojas de apresto sin sentido para el que aprende. Por el contrario, aprender el lenguaje es aprender a dar significado, aprender a darle sentido al mundo; los estudiantes llegan a las aulas con una gran variedad de conocimientos, que se constituyen en un excelente recurso para construir nuevos aprendizajes a partir de acciones contextualizadas y significativas que prepare el docente. En este contexto, se respeta al estudiante, así como su origen y forma de hablar, y se le estima en toda su diversidad.

En este enfoque, el niño y el educador tienen un papel protagónico, ambos son mediadores en los procesos de enseñanza y aprendizaje. El docente debe saber cómo se aprende, cómo se desarrolla el lenguaje y cómo se promueven ambientes que estimulen el aprendizaje; debe estar consciente de que el aprendizaje es primero social y luego individual, que la autonomía se construye, que la afectividad es el motor o freno del desarrollo, que es esencial el respeto hacia los estudiantes y que para que se dé el aprendizaje, éste debe estar centrado en hechos relevantes y significativos (Goodman, 1989).

Las educadoras y los educadores organizan contextos sociales para que sus estudiantes compartan su trabajo en forma oral o escrita, observan el juego y el trabajo de los infantes y comprenden que la acción y el lenguaje que utilizan las niñas y los niños son el reflejo de su cultura y, en consecuencia, es lo verdaderamente significativo para ellos, por lo que, a partir de ahí, se trabaja la zona de desarrollo próximo.

El espacio que se da entre la zona de desarrollo real y el nivel de desarrollo potencial es lo que Vigotsky llama "zona de desarrollo próximo", la cual se define como la distancia entre el nivel de desarrollo real, determinado por la capacidad de resolver problemas de manera independiente, y el nivel de desarrollo potencial, determinado por la capacidad de resolver problemas bajo las orientaciones de un adulto o en colaboración con pares más capacitados (Vigotsky, 1978). 
El educador trabaja la zona de desarrollo próximo ayudando a que el infante logre su desarrollo potencial mediante una oportuna intervención individual o grupal que posibilite el intercambio, la crítica, la discusión y la solución de problemas. En este proceso, el maestro es un mediador que brinda la ayuda necesaria para que el niño y la niña aprendan. (Arellano, 1997 y Goodman, 1993).

Para la filosofía de Lenguaje Integral, la integración curricular es fundamental, pues los procesos de enseñanza y de aprendizaje de la lectoescritura no se dan fraccionados, divididos en contenidos o destrezas, sino de una manera integrada y natural, al igual que se aprende a caminar y a hablar. (Sáez y otras, 1996)

El desarrollo del lenguaje y del contenido se aprenden simultáneamente; de hecho, el docente ofrece oportunidades para que los estudiantes participen en experiencias auténticas de habla, de lectura y de escritura, y por otra parte, investiga sobre los contenidos que las niñas y los niños desean conocer. El contenido del curriculum se desarrolla en torno a los intereses y a las experiencias que los educandos tienen en su vida diaria fuera de la escuela, en su contexto sociocultural, con el propósito de incorporar toda la variedad de funciones lingüísticas orales y escritas (Goodman, 1989).

Con el fin de brindar experiencias auténticas y divertidas de lenguaje, en los centros infantiles se programan diferentes actividades, entre ellas:

- Lectura diaria de cuentos, rimas, poesías, etiquetas, rótulos, entre otros.

- Exposición de los niños y las niñas sobre diferentes temas de su interés.

- Dramatización de cuentos y poesías

- Creación de textos de parte de los párvulos mediante dibujos, letras y símbolos que ellos mismos construyen y leen.

- Planificación de las estrategias de aprendizaje en colaboración los niños y las niñas, las cuales se escriben en la pizarra y posteriormente se leen.

En este contexto, la educadora y el educador deben: crear actividades significativas que estimulen el pensamiento divergente, construir significados y buscar soluciones a las inquietudes que se presentan; pero para ello es necesario "elaborar preguntas constructivas que obliguen al sujeto a reflexionar lógicamente y a inferir situaciones" (Gómez, M; Villareal, B; González, L; López, L y Jarillo, R 1995, p. 43) y ofrecer "...experiencias lingüísticas tan auténticas e importantes como las que ocurren fuera de la escuela, llegar a cada niño $y$ ayudarlo a ampliar su competencia lingüística en la medida que continúa aprendiendo a través del lenguaje" (Goodman, 1993, p. 23). Con actividades auténticas de lectoescritura, se activa en el párvulo el proceso de asimilación y acomodación del lenguaje escrito, al 
enfrentar "contradicciones entre previos esquemas y la nueva experiencia que genera el "desequilibrio". Para resolver estos conflictos, la niña y el niño formulan nuevas hipótesis y acomodan nuevos esquemas lingüísticos que aparentan resolver su problema para usar el lenguaje (Ruiz, 1996, p. 47). En este proceso de asimilación y acomodación, el infante, poco a poco, abstrae las normas del lenguaje alfabetizado convencional (Ferreiro, 1983).

Un salón de clase de educación inicial debe contar con un ambiente letrado, pertinente culturalmente y rico en material impreso que propicie el diálogo, el juego dramático, el dibujo, la creación de textos y actividades de lectura y escritura individuales y colectivas.

Desde este enfoque, la evaluación se constituye en una constante reflexión del trabajo realizado, de los logros obtenidos y de las metas por alcanzar. El educador y la educadora se convierten en creadores de situaciones de aprendizaje y en investigadores de su práctica pedagógica y son conocedores de la importancia de la participación de la familia en el desarrollo de los procesos de aprendizaje del párvulo.

Prácticas pedagógicas fundamentadas en la filosofía del Lenguaje Integral se aplican con éxito en centros infantiles de Venezuela (Arellano, 1994), Puerto Rico (Sáez y otras, 1996; Ruiz.1996), Perú (Camargo y Cartagena, 1999), Estados Unidos (Goodman, 1993), en nuestro país en la Escuela Nueva Laboratorio de la Universidad de Costa Rica y en algunos centros infantiles de educación inicial.

\section{Reflexiones finales}

Las nuevas contribuciones teóricas y prácticas en torno a los procesos iniciales del aprendizaje de la lectura y de la escritura, nos llevan a cuestionar las prácticas pedagógicas que se desarrollan en los centros de educación inicial con el propósito de "preparar" al niño y a la niña para el ingreso a la escuela. Es preciso un cambio educativo que lleve a la transformación de este nivel; para ello, es necesario tener en cuenta los siguientes aspectos:

- El niño y la niña construyen el conocimiento en interacción con el medio físico y social que los rodea, formulan hipótesis y se explican el mundo de acuerdo con sus esquemas mentales, lo que implica que el infante ingresa al centro infantil trayendo conocimientos sobre la lengua escrita, conocimientos que debe tomar en cuenta el docente al planificar las estrategias pedagógicas.

- La niña y el niño construyen y reconstruyen el conocimiento sobre el lenguaje escrito de la misma forma en que construyen el lenguaje oral, en un contexto natural mediante 
experiencias cooperativas, reales, pertinentes y significativas de lectura y escritura. De esta manera, poco a poco, abstraen las normas del sistema fonético, sintáctico y semántico del lenguaje escrito (Ruiz, 1996).

- El lenguaje sirve para organizar el pensamiento, para aprender, comunicar y compartir experiencias con los demás; el desarrollo cognoscitivo, y lingüístico son interdependientes.

- En el aprendizaje de la lecto-escritura interviene en gran medida el contexto sociocultural que rodea al niño y a la niña, por lo tanto, es necesario partir su de cultura, de lo más significativo y cercano a su vida (Peralta, 1993).

- La función social que tiene la lengua escrita para comunicar significados y transmitir conocimientos, creencias y valores, es fundamental para el desarrollo del lenguaje. En una sala de clase de educación inicial, se debe propiciar la participación del niño y la niña en los cuatro procesos del lenguaje: expresión oral, compresión oral, comprensión gráfica, y expresión gráfica "lo que refuerza y refina los esquemas lingüísticos y conceptuales" (Ruiz, 1996, p. 56).

- El infante debe comprender que el lenguaje escrito, al igual que el lenguaje oral, sirve para comunicar ideas y pensamientos, transmite significados y es funcional. Mediante actividades auténticas de lenguaje descubre "...que puede escribir lo que dice, leer lo que escribe y puede ser el autor de su propio libro" (Ruiz, 1996, p. 84).

- El ambiente del centro infantil debe propiciar oportunidades para que el infante dialogue, dibuje, escriba y lea libre y espontáneamente; es decir; juegue con el lenguaje. Para ello, es importante contar con un ambiente letrado, rico en materiales, al alcance de las niñas y los niños, que inviten a la exploración y a la manipulación. Además de las áreas de trabajo que tradicionalmente tiene una sala de clase de educación inicial, es importante organizar un "centro de lenguaje" donde los niños puedan utilizar diversos medios para comunicarse. En condiciones ideales, en este espacio se ubican libros de cuentos, de conceptos, de poesías, de rimas y libros elaborados por los mismos niños y niñas, láminas, títeres, juegos alfabetizados, hojas blancas de diferentes tamaños, papel de construcción, sobres, grapadora, buzón, lápices, marcadores, pizarras, etiquetas de 
productos y letreros conocidos por los niños; si es posible, grabadora y audífonos, casetes con juegos, cuentos y poesías grabadas, y máquinas de escribir, entre otros materiales que incentivan el desarrollo de la expresión oral y escrita. Es necesario que el material y las áreas estén rotuladas por docentes o estudiantes. El material debe estar en buen estado para que los infantes aprendan a cuidarlos y manipularlos adecuadamente; además, estos se deben cambiar periódicamente tomando en cuenta el interés y el uso que se les dé.

- La educadora y el educador deben propiciar mediante el juego cooperativo todo tipo de actividades que incentiven el desarrollo del lenguaje en los párvulos: dramatizaciones, conferencias, elaboración de carteles, cuentos, cartas, mensajes, poesías, canciones, lectura de cuentos y poesías, entre otras estrategias de aprendizaje que se puedan crear en torno al tema de interés que se esté trabajando con los infantes, e integrando los procesos del lenguaje con las áreas curriculares.

- Es preciso que el educador analice y evalúe su práctica pedagógica y la congruencia con las premisas teóricas del constructivismo. Es necesario crear círculos de estudio entre colegas, compartir lecturas, experiencias y promover la investigación de lo que ocurre día a día en el aula.

- Es fundamental que la evaluación se perciba como un proceso continuo de análisis sobre el trabajo cotidiano de los niños y las niñas, por ello, es importante la observación constante y la recopilación y organización de las producciones que permitan hacer juicios sobre el aprendizaje de los infantes. De esta forma, el educador y la educadora podrán detectar el desarrollo real que tienen sus estudiantes e incentivar su desarrollo potencial.

- Para el éxito de un programa inicial de lecto-escritura, es importante la participación de la familia en la sala de clase y en actividades de lectura y escritura propuestas en el hogar, todas con fines prácticos y divertidos.

Para terminar, es preciso aclarar que no se trata de escolarizar el jardín de infantes, se trata de brindarles oportunidades a los niños y a las niñas para que exploren creativamente la escritura y la lectura, y reinventen el lenguaje escrito para que se apropien de él. En síntesis, se trata de que niños y niñas construyan el placer de leer y escribir. 


\section{Referencias}

Akirov, A. (1998). Importancia de las actividades de prelectura en la construcción de significados. En Carolina: Humanismo y Tecnología. San Juan, Puerto Rico: Colegio Regional de Carolina Universidad de Puerto Rico. Número extraordinario: Tercer Congreso de las Américas sobre Lectoescritura. Año III - Núm.3 - enero a julio, pp 94102.

Arellano-Osuna, Adelina. (1989). El lenguaje integral y la lectoescritura en la escuela primaria latinoamericana. En Lectura y Vida. Diciembre. pp. 5-11.

Arellano-Osuna, Adelina. (1994). El Lenguaje integral: una alternativa para la Educación. Mérida: Venezuela: Venezolana C.A. 133 p. amigo.104 p.

Arellano-Osuna, Adelina. (1996). ¿Qué estamos haciendo en América Latina para desarrollar lectores y escritores autónomos? Memoria del Segundo Congreso de las Américas sobre Lectoescritura. Universidad Nacional. Dirección de Extensión. Heredia: EUNA. pp17-22

Arellano-Osuna, A. (1997). Lenguaje integral para leer, escribir y aprender. Lima: Libro.

Barca L., A. y Porto R., A. (1998). Cap. VII Desarrollo y aprendizaje del lenguaje en contextos educativos. En Desarrollo del lenguaje y cognición. Manuel Peraldo y otros: Psicología Pirámide.

Barquero, R. (1996). Vigotsky y el aprendizaje escolar. Buenos Aires, Argentina: Aique $255 \mathrm{p}$.

Camargo, I. y Cartagena. (1999). Lenguaje integral: una experiencia constructivista para el aprendizaje exitoso de la lectoescritura. En Revista Lectura y Vida. Buenos Aires, Argentina. Marzo, pp21-31.

Carretero, Mario. (1998). Cap V. Cuando la mente va a la escuela. En Introducción a la psicología cognitiva. Buenos Aires, Argentina: AIQUE pp. 235-258

Chacón, Nora. (1974). Un criterio ecléctico en la enseñanza de la lectoescritura. San José, Costa Rica: Ed. Fernández Lobo. 95 p.

Chavarría, M. Celina. (1993). Tendencias Políticas de la Educación Preescolar. San José, Costa Rica: Editorial de la Universidad de Costa Rica. p.139

Coll, Cesar y Solé, Isabel. (1998). Los profesores y la concepción constructivista. En El constructivismo en el aula. 8ª edición. Barcelona, España: GRAÓ. pp 7-23

Denies, Cristina de (1989). Didáctica del Nivel Inicial o Preescolar. Buenos Aires: El Ateneo. $165 \mathrm{p}$.

Dengo, María Eugenia. (1998). Educación Costarricense. San José, Costa Rica: EUNED $293 p$.

Fleisher, Carol. (1990). El pensamiento a partir del lenguaje: la construcción lingüística de 
las representaciones cognitivas. En La elaboración del sentido. Compiladores Jerome Bruner y Helen Haste. Barcelona, España: Paidós, pp125-138.

Ferreiro, Emilia y Ana Teberosky. (s.f) (1982). Prólogo e introducción En La teoría de Piaget: Investigaciones en los sistemas de escritura. Siglo XXI pp 10-47.

Ferreiro, Emilia. (1983). Psico-génesis de la escritura. En Psicología genética y aprendizajes escolares. Compilación de Cesar Coll. Barcelona, España: Siglo XXI.

Ferreiro, Emilia. (1997). Alfabetización. Teoría y práctica. México: Siglo Veintiuno, S.A. 202 p.

Flórez, Rafael. (s.f.) Hacia una pedagogía del conocimiento. Santa Fe de Bogotá, Colombia. pp 234-284.

Gómez, Margarita. (1994). La adquisición de la lengua escrita. En Los procesos de lectura y escritura. Selección de lecturas / Com. por Marta Eugenia Sánchez y Luz Emilia Flores. Heredia: EUNA. pp 55-60

Gómez, Margarita, Villarreal, Beatriz, González, Laura; López, Lourdes y Jarillo, Remigio (1995). El niño y sus primeros años en la escuela. México: SEP.229 p

Goodman, Kenneth. (1989). Lenguaje Integral. Mérida, Venezuela: Editorial Venezolana. $115 \mathrm{p}$.

(1993). El Lenguaje Integral como Filosofía Educativa. En Memoria del Primer Congreso de las Américas sobre Lectoescritura 1992. Maracaibo, Venezuela: Universidad de los Andes. pp 4-29

Goodman, Kenneth y Yeta Goodman. (1993). Vygotsky desde la perspectiva del lenguaje total. En Vygotsky y la educación. $2^{a}$ Edición. Buenos Aires, Argentina: Aique pp. 263292.

Halliday, M. A. K. (1986). El lenguaje como semiótica social. México: Fondo de cultural económica $327 \mathrm{p}$.

Larrauri, R. (1998). Los niños pueden "leer" y "escribir" con placer desde la Educación Inicial. En Carolina: Humanismo y Tecnología. San Juan, Puerto Rico: Colegio Regional de Carolina Univ. de Puerto Rico. Número extraordinario: Tercer Congreso de las Américas sobre Lectoescritura. Año III - Núm.3 - enero a julio. pp 66-70.

Luria, A. R. (1985). Lenguaje y pensamiento. 2ª Edición. Barcelona, España: Martínez Roca S. A. $160 p$

Luria, A. (s.f.) Vygosky y las funciones psíquicas superiores. San José, Costa Rica: Proyecto SIMED.

Matos, J. (1996). El paradigma sociocultural de L. S. Vigostky y su aplicación en la educación. (mimeo). Heredia, Costa Rica: Universidad Nacional. 13 p. 
Ministerio de Educación Pública. (1990). Política Curricular. San José, Costa Rica: MEP $44 \mathrm{p}$.

Ministerio de Educación Pública. (1996). Programa de Estudio del Ciclo de Transición de la Educación Preescolar. San José, Costa Rica: MEP.

Molina, Ángeles. (1998). Reseña del libro La alfabetización temprana en el ambiente preescolar: Nuevas perspectivas para leer y escribir, de Daisy Ruiz. Pedagogía. 32. San Juan Puerto Rico: Recinto de Río Piedras, Facultad de Educación. Universidad de Puerto Rico $108-112$.

Moll L. (1993). Vygotsky y la educación. 2a Edición. Buenos Aires: Aique, 493 p Puerto Rico.

Montessori, M. (1939). Manual Práctico del Método Montessori. $3^{a}$ Edición. Barcelona España: Casa Editorial ARALUCE. 167p

Morales, P. (1990). El papel del lenguaje en el desarrollo cognoscitivo: anteposición de la perspectiva piagetiana frente a la perspectiva soviética (mimeo). Puerto Rico: Recinto de Río Piedras, Facultad de Educación. Universidad de Puerto Rico.

Mota, C. (1998). Innovaciones creativas en Lecto-escritura. En Carolina: Humanismo y Tecnología. San Juan, Puerto Rico: Colegio Regional de Carolina Universidad de Puerto Rico. Número extraordinario: Tercer Congreso de las Américas sobre Lectoescritura. Año III - Núm.3 - enero a julio.

Myers, Robert. (1995). La educación Preescolar en América Latina. El Estado de la Práctica. Santiago de Chile. Programa de Promoción de la Reforma Educativa en América Latina. 31p.

Onrubia, Javier. (1998). Enseñar: crear zonas de desarrollo próximo e intervenir en ellas. En El constructivismo en el aula. 8ª edición. Barcelona, España: GRAÓ, pp 101-123.

Papalia, Diane y Wendkos, Sally. (1997). Psicología del Desarrollo. $7^{\text {a }}$ Edición. Santa Fé de Bogotá, Colombia: McGraw Hill. 671p.

Peralta, Victoria. (1993). El currículo en el jardín infantil. Santiago de Chile: Andrés Bello.324 p.

Rivera, José. (1998). La educación infantil en el siglo XXI. Proyecto Principal de Educación en América Latina y el Caribe. Boletín 47. Santiago de Chile: UNESCO, Oficina Regional de Educación para América Latina y el Caribe. pp.41-72

Rivière, A. (1988). La psicología de Vygotski. 3ª Edición. Madrid: Visor. 100 p.

Rodríguez, Wanda. (1998). La perspectiva constructivista en la educación. Pedagogía, 32. San Juan Puerto Rico: Recinto de Río Piedras, Facultad de Educación. Universidad de Puerto Rico 6-20.

Rojas, Maricela. (1998). Educación Científica y Matemática para el niño preescolar I. Perspectiva Constructivista. San José, Costa Rica: EUNED. 171 p. 
Ruiz, Daisy. (1996). La alfabetización temprana en el ambiente preescolar. San Juan, Puerto Rico: Facultad de Educación, Universidad de Puerto Rico. Recinto Río Piedras. $192 \mathrm{p}$.

Sáez, Ruth. (1996ª). ¡Claro que somos autores! La autenticidad en la escritura temprana. En Memoria del Segundo Congreso de las Américas sobre Lectoescritura. Universidad Nacional. Dirección de Extensión. Heredia: EUNA pp 265-269

Sáez, Ruth y otras. (1996). El lenguaje integral y la integración curricular: la experiencia de un kindergarten puertorriqueño. En Memoria del Segundo Congreso de las Américas sobre Lectoescritura. Universidad Nacional. Dirección de Extensión. Heredia: EUNA pp 339-346

Sánchez, M. E.; Castro, M.; Vargas, M. (1996). La importancia de la atención de los procesos iniciales de lectura y escritura en la formación de niños productores y lectores de textos. En Memoria del Segundo Congreso de las Américas sobre Lectoescritura. Universidad Nacional. Dirección de Extensión. Heredia: EUNA pp 271276.

Solís, Virginia. (1992). El curriculum preescolar costarricense. Memoria I Simposio de Educación Preescolar. San José Costa Rica: Departamento de Educación Primaria y Preescolar. Escuela de Formación Docente. Facultad de Educación. Universidad de Costa Rica. pp. 26-39.

Torres Santomé, J. (1996). Globalización e interdisciplinariedad: el curriculum integrado. $2^{a}$ Edición. Madrid, España: Morata 279 p.

Ugalde, M. E. (1983). Principios y técnicas de la Educación Preescolar. San José, Costa Rica: EUNED. 312 p.

Vigotsky, L. S. (1978). Pensamiento y Lenguaje. Buenos Aires, Argentina: La Pleyade 220 p

Weaver, Constance. (1990). Understanding Whole Language. Toronto: Irwin Publishing. $309 \mathrm{p}$. 